\title{
A broadband quad-stable energy harvester and its advantages over bi-stable harvester: Simulation and experiment verification
}

Zhiyong Zhou ${ }^{1)}$ Weiyang Qin ${ }^{1)^{*}}$ Pei Zhu ${ }^{1)}$

1) (Department of Engineering Mechanics, Northwestern Polytechnical University, Xi'an 710129, People's Republic of China)

\begin{abstract}
This paper presents a novel quad-stable energy harvester (QEH) to harvest vibration energy. The QEH is composed of a piezoelectric bimorph cantilever beam with a tip magnet and three external fixed magnets. The potential energy of the QEH is derived, which has four potential wells and three low barriers. This implies that the QEH needs relatively lower excitation energy to cross the barriers than the bi-stable energy harvester (BEH). Simulations are carried out for the BEH and QEH at different levels of excitation. Results show that the QEH owns a smaller threshold and wider range of frequency for occurrence of snap-through than the $\mathrm{BEH}$. Thus the QEH can give out a large output voltage. Corresponding experiments were performed for validation. The experimental results are in agreement with the simulations, which means that the QEH can improve the harvesting efficiency considerably.
\end{abstract}

Keywords: piezoelctric bimorph, quad-stable energy harvester, snap-through

\section{Introduction}

Energy harvesting from ambient vibration power in the environment has been intensively studied during the past few years, mainly because of the need to power wireless sensors or communication node networks. The methods of transduction of vibration to electricity via piezoelectric materials have motivated many researchers because of its high power density, green and high voltage output properties [1-5]. The typically piezoelectric harvester is composed of a cantilever beam with one or more piezoelectric patches which can generate electric charge when subjected to mechanical strain.

The linear devices can only exhibit resonance within a very narrow frequency

\footnotetext{
*Corresponding author:

Weiyang Qin, Department of Engineering Mechanics, Northwestern Polytechnical University, Xi' an 710129,

People's Republic of China

Email: qinweiyang@ aliyun.com (Weiyang Q.)

Tel. 086-15229030828
} 
range. To make them more applicable researchers began to explore active and semiactive strategies to increase their efficiency [6-8]. Within the past few years, the bistable piezoelectric inertial generator has emerged as a popular mechanism for harvesting. The main advantage of nonlinear energy harvesters over their linear counterparts is that the nonlinear harvesters scavenge energy over a broader frequency range of vibrations. It has been demonstrated that nonlinear bi-stable systems, under proper conditions, have better performance in terms of the amount of energy extracted from wide spectrum vibrations [9-14]. A typical BEH is as shown in Fig. 1, which has two stable equilibrium positions and an unstable equilibrium position.

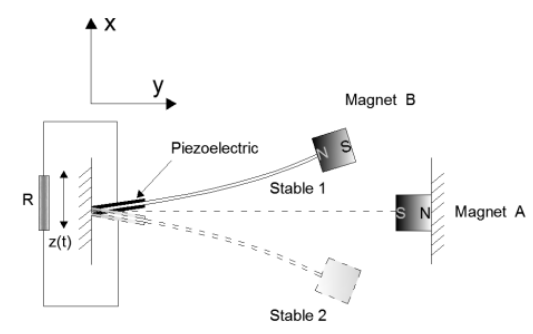

Fig. 1. Bi-stable energy harvester (BEH) with two stable states.

To enhance the conversion ability of the piezoelectric energy harvester, magnets have been frequently adopted to alter the stiffness of the energy harvester and thus tune the resonant frequency of the energy harvester, which is ideally suited to efficiently harvest energy from ambient excitations with slowly varying frequencies $[15,16]$. Tang [17] exploited a pair of magnets in repulsive and attractive configurations to enhance the vibration amplitude and the bandwidth of frequency. Miso et al. [18] presented a closed form solution to harvesting efficiency that allows device comparison. The optimized electrical loading conditions are studied. The BEH device has the capability of snapping from one stable state to another at a certain intensity of excitation. Such snap-through motion can bring about a large-amplitude vibration and shows a good performance in energy harvesting. But there exists a difficult point for the bi-stable harvester. The increase of the distance between the two potential wells can enlarge the amplitude of snap-through, but the barrier height will increase accordingly. This hinders the BEH from breaking through the constraint of potential barriers [19].

To further enhance the performance of the energy harvester, some studies focused on using adjustable or movable magnets. Lin [20] investigated a magnetically coupled piezoelectric cantilever beam, in which the displacement of the fixed magnet could be altered to achieve off-resonance to enhance the broadband frequency response. Zhou et al. [21] investigated a magnetically coupled nonlinear piezoelectric energy harvester by altering the angular orientation of its external magnets for enhanced broadband frequency response. Tang and Yang [22] introduced a magnetic coupled piezoelectric 
energy harvester in which the magnetic interaction is produced by a magnetic oscillator. Gao et al. [23] conceived a structure with an elastic support external magnet and proved that elastic support systems have better power output performance than rigid support systems when excited at low-intensity vibrations. However, when it comes to small excitations, the above improved BEH cannot gain enough energy to break through the constraint of potential wells and generally go to intrawell oscillations. So the performance of the bi-stable energy harvester is not satisfied at all time.

Recently, more complex energy harvesting systems with multi-stable state have been proposed. Jung et al. [24] investigated the piezoelectric energy harvester with two rotatable external magnets. The results show that the angle of the external magnet and separation distance between the tip magnet and the external ones could alter the potential energy of the harvester system. Kim and Seok [25] investigated the numerical analysis of bifurcation of a multi-stable bimorph energy harvester that used the magnetic attraction effect. Zhou et al. [26, 27] carried out numerical and experimental investigations on a tri-stable bimorph cantilever energy harvester with two rotatable external magnets at swept sine excitations. The results reveal that the tri-stable energy harvester can improve the broadband performance to generate high energy output. Cao et al. [28] considered the influence of potential well depth on tri-stable energy harvesting performance and the corresponding experiment results show that the shallower potential well depth will enhance the broadband performance and the capability of harvesting energy at low frequency excitations. Tékam et al. [29] analyzed the dynamics of a tri-stable energy harvester which has fractional order viscoelastic flexible material and the results showed that the energy output could be enhanced by choosing a material with a small value of viscoelasticity coefficient and a large fractional order.

This paper proposes a novel quad-stable energy harvester (QEH). The theoretical model of the QEH is established using energy-based methods and Hamiltonian principle. The potential energy of the BEH and QEH are derived. The electromechanical equation is solved and the results show that the QEH owns a wide frequency bandwidth for occurrence of snap-through. Compared with the BEH, the QEH can realize snap-through more easily and generate high output voltages.

\section{Quad-stable piezoelectric energy harvester}

As Fig. 2 shows, the QEH consists of a bimorph cantilever beam with a tip magnet $\mathrm{D}$ and three fixed magnets A, B and C. Two piezoelectric layers are attached to the root of the cantilever beam. The piezoelectric layers are connected to an electrical load. 


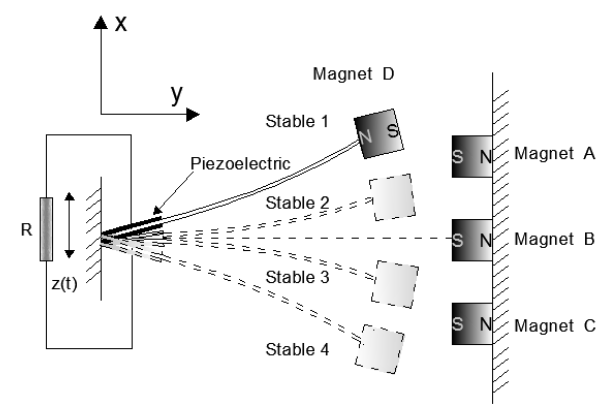

Fig. 2. Quad-stable cantilever piezoelectric energy harvester.

The dynamic equations of the QEH can be derived by the Euler-Bernoulli beam theory and Hamilton principle. The general form of Hamilton's principle for this electromechanical system can be written as

$$
\int_{t_{1}}^{t_{2}}\left(T_{b}+T_{M}-U_{b}-U_{m}\right)=0
$$

where $T_{b}$ is the kinetic energy of the substrate and piezoelectric laminates, and $U_{b}$ is its potential energy; $T_{M}$ is the kinetic energy of the end mass (magnet $\mathrm{D}$ ), and $U_{m}$ is the potential energy that is produced by interactions between the external magnets (A, $\mathrm{B}$ and $\mathrm{C}$ ) and magnet $\mathrm{D}$.

The kinetic energy $T_{b}$ can be evaluated by

$$
T_{b}=\frac{1}{2} \rho_{S} b_{S} h_{S} \int_{0}^{L}\left(\frac{\partial w(x, t)}{\partial t}+\dot{z}(t)\right)^{2} d x+\frac{1}{2} \rho_{p} b_{p} h_{p} \int_{0}^{L_{p}}\left(\frac{\partial w(x, t)}{\partial t}+\dot{z}(t)\right)^{2} d x,
$$

where the overdot denotes the derivative with respect to time; $L$ is the length of the piezoelectric beam; $w(x, t)$ denotes the transverse displacement, and $z(t)$ is the base displacement; $b_{S}$ and $h_{S}$ are the width and thickness of substrate respectively; $\rho_{S}$ and $\rho_{p}$ are the densities of the substrate and piezoelectric materials respectively; $b_{p}$ and $h_{p}$ are the width and thickness of piezoelectric layer respectively, and $l_{p}$ is its length.

The kinetic energy $T_{M}$ is

$$
T_{M}=\frac{1}{2} M_{t}\left(\left.\frac{\partial w(x, t)}{\partial t}\right|_{x=L}+\dot{z}(t)\right)^{2}+\frac{1}{2} I_{t}\left(\left.\frac{\partial^{2} w(x, t)}{\partial t \partial x}\right|_{x=L}\right)^{2},
$$

where $M_{t}$ is the mass of the magnet $\mathrm{D}, I_{t}$ is the rotational inertia of the end mass (magnet D).

The potential energy $U_{b}$ can be given by

$$
\begin{gathered}
U_{b}=\frac{1}{2} E I \int_{0}^{L}\left(\frac{\partial^{2} w(x, t)}{\partial x^{2}}\right)^{2} d x+C_{11}^{E} I_{p} \int_{0}^{l_{p}}\left(\frac{\partial^{2} w(x, t)}{\partial x^{2}}\right)^{2} d x-\vartheta_{p} v(t)\left(h_{S}+\right. \\
\left.h_{p}\right)\left.\frac{\partial w(x, t)}{\partial x}\right|_{x=l_{p}}-\frac{1}{4} C_{p} v^{2}(t),
\end{gathered}
$$


where $v(t)$ is the voltage across electrodes; $I_{p}$ is the moment of inertia of the piezoelectric beam; $E I$ is the bending stiffness of the substrate; the electromechanical coupling term $\vartheta_{p}$ is

$$
\vartheta_{p}=e_{31} E_{p} h_{p} l_{p}\left(h_{s}^{2}-h_{p}^{2}\right) / h_{p}
$$

and the capacitance through the piezoelectric layers $C_{p}$ is

$$
C_{p}=\varepsilon_{33}^{S} b_{p} l_{p} / h_{p}
$$

The transverse deflection $w(x, t)$ can be approximated by the first linear mode of beam as

$$
\begin{aligned}
& w(x, t)=\phi(x) q(t), \\
& \phi(x)=\left\{\begin{array}{l}
\phi_{1}(x), 0 \leq x<l_{p} \\
\phi_{2}(x),
\end{array} l_{p} \leq x \leq L,\right. \\
& \phi_{1}(x)=C_{1} \cos \left(\beta_{1} x\right)+C_{2} \sin \left(\beta_{1} x\right)+C_{3} \cosh \left(\beta_{1} x\right)+C_{4} \sinh \left(\beta_{1} x\right), \\
& \phi_{2}(x)=P_{1} \cos \left(\beta_{2} x\right)+P_{2} \sin \left(\beta_{2} x\right)+P_{3} \cosh \left(\beta_{2} x\right)+P_{4} \sinh \left(\beta_{2} x\right),
\end{aligned}
$$

where $\phi_{1}(x)$ and $\phi_{2}(x)$ represent the first linear mode shapes of the beams with and without piezoelectric layers, respectively; $C_{k}$ and $P_{k}(k=1,2,3,4)$ are constant coefficients derived from the boundary condition; $\beta_{1}$ and $\beta_{2}$ are the eigenvalues of characteristic equation.

In calculating magnetic potential energy, magnets can be modeled as point dipoles [30-32]. The geometric configuration of the tip magnet and the three external magnets is depicted in Fig. 3.

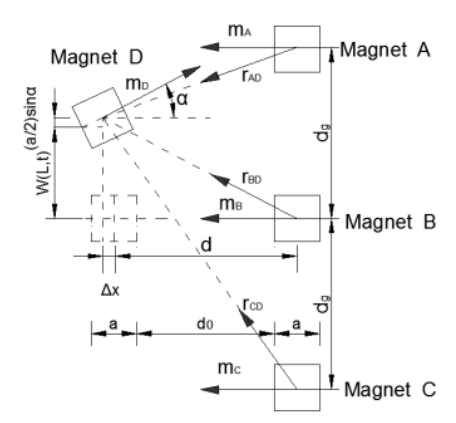

Fig. 3. Geometric configuration of the tip magnet and three external permanent magnets.

The magnetic field generated by magnet $n(n=\mathrm{A}, \mathrm{B}$ or $\mathrm{C})$ upon magnet $\mathrm{D}$ can be evaluated by

$$
\boldsymbol{B}_{n D}=-\frac{\mu_{0}}{4 \pi} \nabla \frac{\boldsymbol{m}_{n} \cdot \boldsymbol{r}_{n D}}{\left\|\boldsymbol{r}_{n D}\right\|_{2}^{3}}
$$


where $\boldsymbol{r}_{n D}$ is the vector directed from the magnetic moment source of magnet $n$ to that of magnet $\mathrm{D} ;\|\|_{2}$ and $\boldsymbol{\nabla}$ denote L2-norm and vector gradient operator, respectively; $\boldsymbol{m}_{n}$ denote the magnetic moment vectors of magnet $n$ ( $n=\mathrm{A}, \mathrm{B}$ or $\mathrm{C}$ ); $\boldsymbol{\mu}_{0}=4 \pi \times 10^{-7} \mathrm{Hm}^{-1}$ is the magnetic permeability constant. Therefore, the potential energy of magnetic field generated by the three external magnets (A, B and C) upon magnet $\mathrm{D}$ can be given by

$$
U_{m}=U_{m A D}+U_{m B D}+U_{m C D}=-\boldsymbol{B}_{A D} \cdot \boldsymbol{m}_{D}-\boldsymbol{B}_{B D} \cdot \boldsymbol{m}_{D}-\boldsymbol{B}_{B D} \cdot \boldsymbol{m}_{D} .
$$

The potential energy between magnet $n$ and D can be calculated [33] and the corresponding expression is

$$
U_{m n D}=\frac{\mu_{0} M_{D} V_{D} M_{n} V_{n}\left(-\left(w(L, t)-D_{n}\right)^{2}+2 d^{2}-3 d\left(w(L, t)-D_{n}\right) w^{\prime}(L, t)\right)}{4 \pi \sqrt{\left(\left(w^{\prime}(L, t)\right)^{2}+1\right)}\left(\left(w(L, t)-D_{n}\right)^{2}+d^{2}\right)^{5 / 2}},
$$

where $w(L, t)$ denote the transverse deflection of the beam's tip at an instant of time $t ; M_{n}$ and $V_{n}$ represent the magnetization intensity and the volume of magnet $n$, respectively; $D_{n}(n=\mathrm{A}, \mathrm{B}$ or $\mathrm{C})$ represents the coordinate of magnet $n$ in $x$-direction $\left(D_{A}=d_{g}, D_{B}=0, D_{c}=-d_{g}\right)$;

The Lagrange function for the system is

$$
\mathcal{L}(x, t)=T_{b}+T_{M}-U_{b}-U_{m}
$$

Applying the mode expansion, substituting Eq. (7) into Eq. (14) and integrating with respect to $x$ gives

$$
\begin{aligned}
\mathcal{L}(t)= & \frac{1}{2} \bar{M} \ddot{q}(t)^{2}+\frac{1}{2} \dot{q}(t) \bar{M} \omega^{2}+\theta v(t) q(t)+\Gamma \dot{q}(t) \dot{z}(t)+ \\
& \frac{1}{4} C_{p} v(t)^{2}+\frac{1}{2} \bar{M} \dot{z}(t)^{2}-U_{m}
\end{aligned}
$$

where $\omega$ is the first natural frequency, $\zeta$ is the corresponding modal damping ratio, and

$$
\begin{aligned}
& \theta=\frac{1}{2} b e_{31} \phi_{1}^{\prime}\left(l_{p}\right)\left(h_{S}+h_{p}\right), \\
& \Gamma=\left(2 \rho_{p} A_{p}+\rho_{S} A_{S}\right) \int_{0}^{l_{p}} \phi_{1}(x) d x+\rho_{S} A_{S} \int_{l_{p}}^{L} \phi_{2}(x) d x+M_{t} \phi_{2}(L), \\
& \bar{M}=\rho_{S} A_{S} L+2 \rho_{p} A_{p} l_{p}+M_{t} .
\end{aligned}
$$

The non-conservative virtual works done by the dissipative and the electric forces can be expressed as

$$
\delta W=-\int_{0}^{L} c \dot{w}(x, t) w \delta(x, t) d x+Q(t) \delta v,
$$


where $c$ is the mechanical damping coefficient and $Q(t)$ is the electric charge passing through the resistive load $R$, and $\dot{Q}(t)=v / R$.

The dynamical equations of the system can be derived by

$$
\begin{aligned}
& \frac{d}{d t}\left(\frac{\partial \mathcal{L}}{\partial \dot{q}}\right)-\left(\frac{\partial \mathcal{L}}{\partial q}\right)=\frac{\delta W}{\delta q}, \\
& \frac{d}{d t}\left(\frac{\partial \mathcal{L}}{\partial \dot{v}}\right)-\left(\frac{\partial \mathcal{L}}{\partial v}\right)=\frac{\delta W}{\delta v} .
\end{aligned}
$$

As a consequence we have

$$
\begin{aligned}
& \bar{M} \ddot{q}(t)^{2}+2 \zeta \omega \dot{q}(t)+\bar{M} \omega^{2} q(t)^{2}-\theta v(t)+g(q(t))=-\Gamma \ddot{z}(t), \\
& \theta \dot{q}(t)+\frac{1}{2} C_{p} \dot{v}(t)-v(t) / R=0,
\end{aligned}
$$

where $\zeta=\mathrm{c} \int_{0}^{L} \phi(x)^{2} d x$ and $g(q(t))=\frac{\partial U_{m}}{\partial q(t)}$. By solving Eqs. (22) and (23), we can acquire the dynamic response and output voltage of the harvesting system.

\section{Simulations and analysis}

In this section, numerical methods are employed to solve the dynamical equations and exhibit the characteristics of the QEH and $\mathrm{BEH}$ at harmonic base excitations. The geometric, material, electromechanical and magnetic parameters of the harvesters (as shown in Fig. 1 and Fig. 2) are given in Table 1.

\section{Table 1}

Model parameters used for numerical and experimental study.

\begin{tabular}{lll}
\hline Symbol & Parameter & Value \\
\hline $\mathrm{L}$ & Length of the substrate of beam & $130 \mathrm{~mm}$ \\
$b_{S}$ & Width of the substrate of beam & $8 \mathrm{~mm}$ \\
$h_{S}$ & Thickness of the substrate of beam & $0.12 \mathrm{~mm}$ \\
$\rho_{S}$ & Substrate material density of the substrate & $7800 \mathrm{~kg} / \mathrm{m}^{3}$ \\
$\zeta$ & Modal damping ratio of the beam & 0.01 \\
$E_{S}$ & Young's modulus of the substrate of beam & $205 \mathrm{Gpa}$ \\
$l_{p}$ & Length of piezoelectric layer & $5 \mathrm{~mm}$ \\
$b_{p}$ & Width of piezoelectric layer & $5 \mathrm{~mm}$ \\
$h_{p}$ & Thickness of piezoelectric layer & $0.15 \mathrm{~mm}$ \\
$\rho_{p}$ & Substrate material density of piezoelectric layer & $1785 \mathrm{~kg} / \mathrm{m}^{3}$ \\
$E_{p}$ & Young's modulus of piezoelectric layer & $2 \mathrm{Gpa}$ \\
$e_{31}$ & Coupling coefficient of piezoelectric layer & $23 \times 10^{-10} \mathrm{C} / \mathrm{N}$ \\
$\varepsilon_{33}^{S}$ & Permittivity constant of piezoelectric layer & $1.06 \times 10^{-10} \mathrm{~F} / \mathrm{m}$ \\
$M_{t}$ & Mass of magnet (A, B, C or D) & $2.8 \mathrm{~g}$ \\
$A$ & Area of magnet (A, B, C or D) & $10 \times 10 \mathrm{~mm}$
\end{tabular}




\begin{tabular}{lll}
$a$ & Thickness of magnet (A, B, C or D) & $5 \mathrm{~mm}$ \\
$\boldsymbol{B}_{r}$ & Residual flux density of magnet (A, B, C or D) & $1.48 \mathrm{~T}$ \\
$d_{g}$ & Value of coordinate of magnets (A, C) in $y$-direction & $25 \mathrm{~mm}$ \\
\hline
\end{tabular}
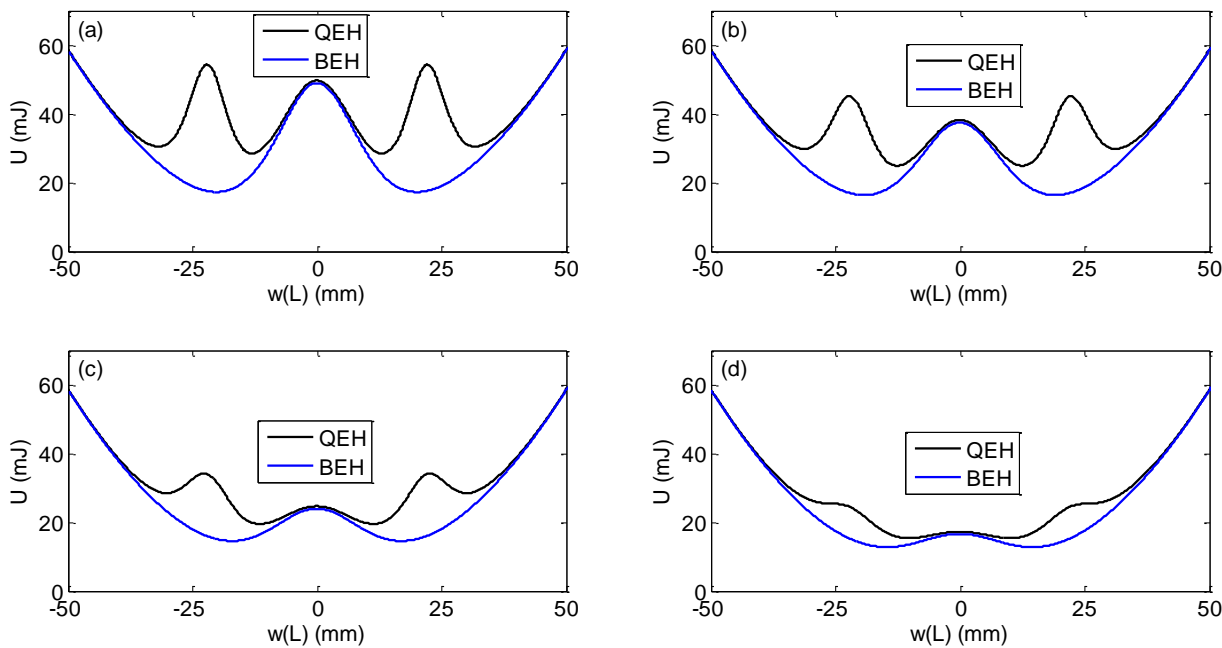

Fig. 4. Potential energy of QEH and BEH for different separation distances: (a) $d_{0}=3.5 \mathrm{~mm}$; (b) $d_{0}=5 \mathrm{~mm}$; (c) $d_{0}=7 \mathrm{~mm}$; and (d) $d_{0}=9 \mathrm{~mm}$.

Recall that from Eq. (12), the magnetic potential energy of the QEH can be calculated. We can carry out a comparison between the potential energies of the QEH and $\mathrm{BEH}$ before solving their electromechanical equations. In practice the potential energies of both the QEH and the BEH consist of two components: the beam elastic potential energy and the magnetic one. Fig. 4 shows the functions of the total potential energies of the QEH and the BEH for different separation distances $\left(d_{0}=3.5 \mathrm{~mm}, 5.0\right.$ $\mathrm{mm}, 7.0 \mathrm{~mm}$ and $9.0 \mathrm{~mm}$ ). It can be seen that the BEH has two potential wells and one barrier between them. The QEH has four potential wells and three barriers between them. It is evident that the barriers of the QEH are lower than those of the BEH for identical separation distance. This implies that the QEH needs relatively lower excitation energy to cross potential barriers than the BEH. Therefore, for small excitation the QEH will exhibit a better performance.
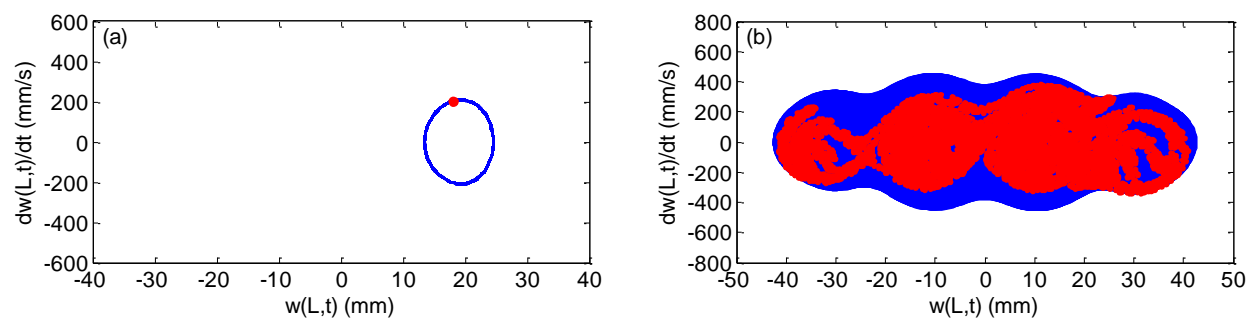

Fig. 5. Phase portrait and Poincare map of the motions at $a_{b}=0.7 \mathrm{~g}$ and $f=8 \mathrm{~Hz}$ : (a) BEH; (b) QEH. 
In order to show their nonlinear characteristics we consider the case of excitation by a harmonic force. The separation distance $\left(d_{0}\right)$ is designated as $5 \mathrm{~mm}$ for both harvesters in the following numerical simulations. First the harmonic base excitation is set $a_{b}=0.7 \mathrm{~g}$ and $f=8 \mathrm{~Hz}$. At this excitation level, it turns out that the BEH can't cross its barrier and is confined in potential wells (Fig. 5(a)). In contrast now the QEH can elicit snap-through and takes an interwell motion, which exhibits a chaotic strange attractor in Poincare map (Fig. 5(b)).
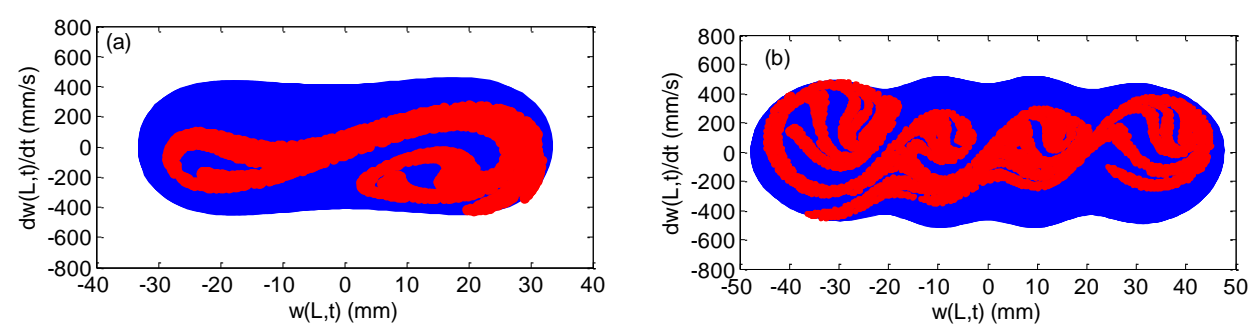

Fig. 6. Poincare map and phase portrait of responses at $a_{b}=1.0 \mathrm{~g}$ : (a) BEH; (b) QEH.

Then the excitation strength is increased to $a_{b}=1.0 \mathrm{~g}$. Fig. 6 shows the phase portrait and Poincare map of the response. At this level the response of the BEH begins to cross the potential barrier and start an interwell motion surrounding the two equilibrium positions (Fig. 6(a)). Meanwhile the QEH arrives at the state of interwell oscillation surrounding the four equilibrium positions. Thus the maximal amplitude of the QEH can reach $48 \mathrm{~mm}$, whereas that of the BEH is merely $32 \mathrm{~mm}$. It should be noted that both responses form a strange attractor in their Poincare maps.
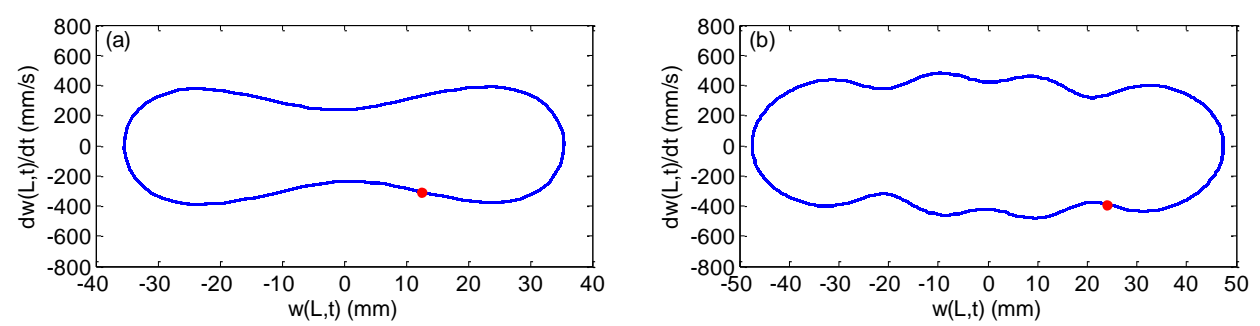

Fig. 7. Poincare map of the motion on its phase portrait for acceleration value $a_{b}=1.0 \mathrm{~g}$ and frequency at $11 \mathrm{~Hz}$ : (a) BEH; (b) QEH.

To see the difference between their amplitudes clearly, we change the excitation frequency a little to $f=11 \mathrm{~Hz}\left(a_{b}=1.0 \mathrm{~g}\right)$. At this frequency both the $\mathrm{BEH}$ and the QEH will exit chaos and go to periodic motions (Fig. 7). From the phase portraits, we can see that both periodic motions circulate their stable equilibrium positions. But the QEH has four stable equilibrium positions, and thus its displacement is much larger than that of the BEH. 

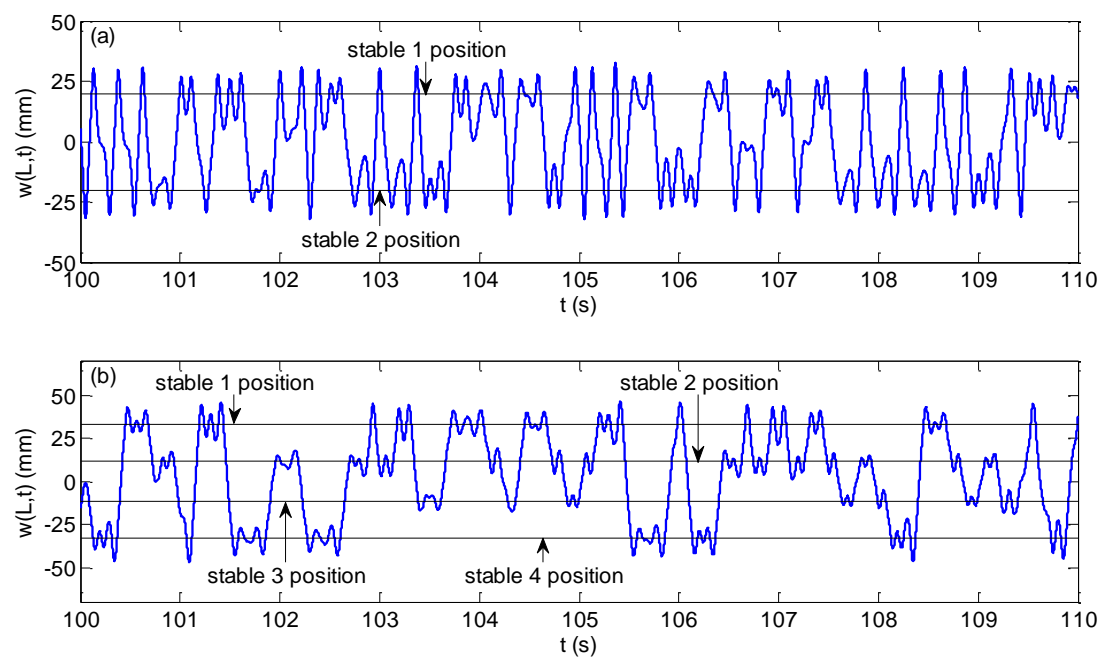

Fig. 8. Numerical results of tip displacement responses of snap-through oscillators for acceleration value $a_{b}=1.0 \mathrm{~g}$ and frequency at $8 \mathrm{~Hz}$ : (a) $\mathrm{BEH}$; (b) QEH.

In order to further study the snap-through phenomenon in the QEH and the BEH, we choose a period of chaos responses of them (Fig. 8). As shown in Fig. 8(a), the BEH's response jumps between its two potential wells at snap-through. The feature of the BEH's snap-through is that its response oscillates at an equilibrium position for a while first and then starts jumping, and there exists a time interval between two jumps. From Fig. 8(b) we can see that the QEH's response can jump between the first and the second positions, the first and the third ones, the second and the third ones, and even the first and the fourth ones at snap-through. Due to the existence of multi-stable positions and the low barriers, the QEH's jump occurs more easily and frequently, so there appears a dense jump in its response. The snap-through between the nonadjacent stable positions brings about a large jump amplitude, which can lead to a high output voltage.
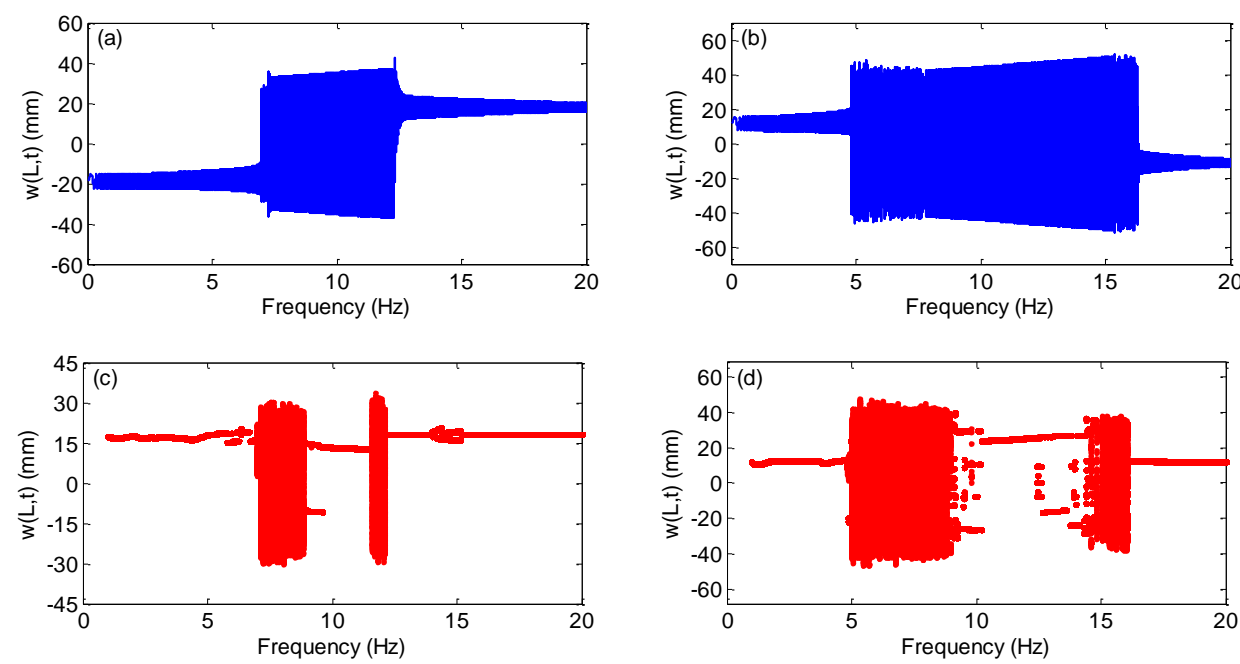

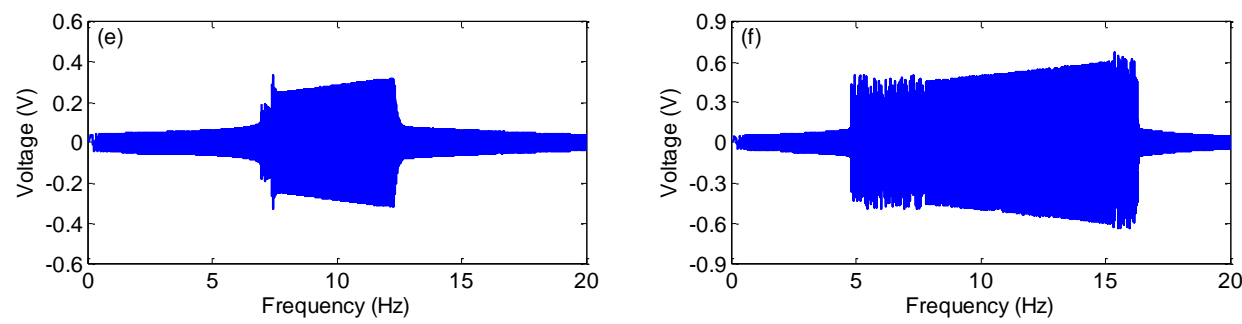

Fig. 9. Simulation responses at frequency sweeping excitation and bifurcation diagrams at different frequencies with $a_{b}=1.0 \mathrm{~g}$ : (a, c, e) BEH; (b, d, f) QEH.

In order to compare the frequency ranges for occurrence of snap-through in the QEH and BEH, we carry out simulations of frequency sweeping for them. The sweeping range is set $0-20 \mathrm{~Hz}$ and the acceleration amplitude is $a_{b}=1.0 \mathrm{~g}$. The results of displacement, bifurcation diagrams and voltage are shown in Fig. 9. For a certain range of frequency the snap-through between potential wells can appear in both experiments of the $\mathrm{BEH}$ and $\mathrm{QEH}$, but the difference is obvious. The QEH can elicit snap-through over nearly a whole range of $5-16.8 \mathrm{~Hz}$, whereas the BEH only has snapthrough within 6.8-12.3 Hz. From the bifurcation diagrams shown in Fig. 9 (c, d), we can distinguish the types of jumping. As to output voltages, the QEH can give out a higher RMS voltage in a much wider bandwidth than the BEH (Fig. 10). There is a large distance between their highest RMS voltages as well in Fig.10. For example, at $a_{b}=0.7 \mathrm{~g}$, the highest RMS voltage generated by the QEH can reach $0.43 \mathrm{~V}$, whereas that generated by the $\mathrm{BEH}$ is only $0.28 \mathrm{~V}$. Therefore, the $\mathrm{QEH}$ can harvest excitation energy more effectively than the BEH.
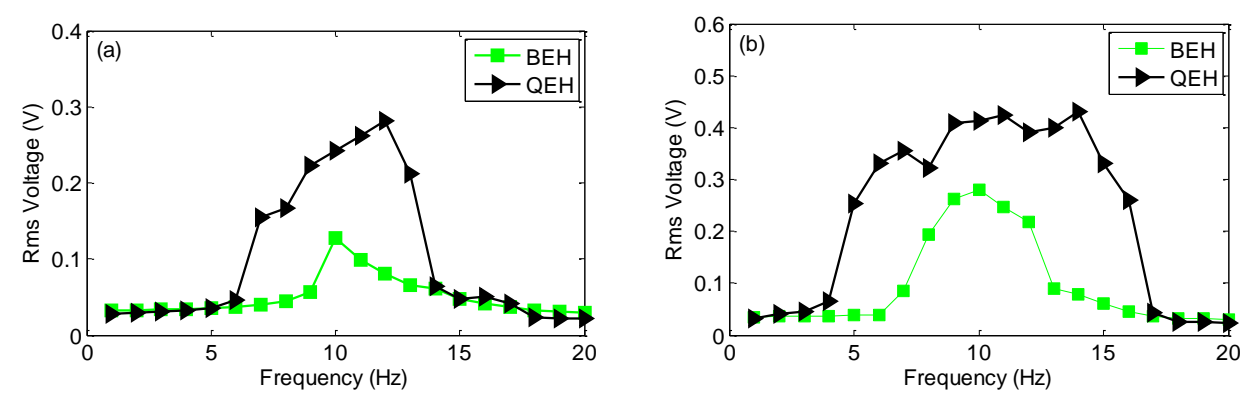

Fig. 10. Variation of RMS voltages with excitation frequency: (a) $a_{b}=0.7 \mathrm{~g}$; (b) $a_{b}=1.0 \mathrm{~g}$.

\section{Validation experiments and results}




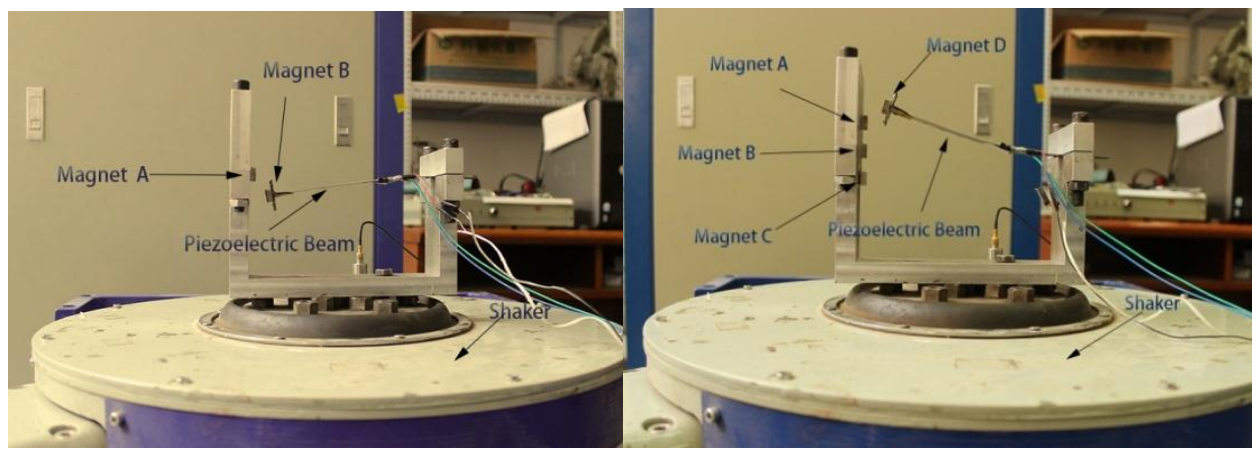

(a) $\mathrm{BEH}$

(b) $\mathrm{QEH}$

Fig. 11. Prototypes of the BEH and QEH.

In order to validate the theoretical analysis, corresponding experiments were designed and carried out for the BEH and QEH. Their experimental setups are as shown in Fig. 11. They both consist of a bimorph piezoelectric beam with a tip magnet, a fixture and several fixed magnets. The BEH has one fixed magnet (A), while the QEH has three fixed magnets (A, B and C). As for the piezoelectric beam, its substrate layer is made of stainless steel, on which two piezoelectric patches were bonded. The QEH's four neodymium magnets have identical dimensions; the edge distance between any two magnets are set identical $(10 \mathrm{~mm})$, then the distance between the centers of them is $20 \mathrm{~mm}$, which is designed to ensure the occurrence of four equilibrium positions. On the other hand an accelerometer was mounted on the top of the shaker to monitor its acceleration, and a signal measurement device (DH5922) was used to record output voltages and strains. The base acceleration $\left(a_{b}\right)$ was designated as $1.0 \mathrm{~g}$ ( $\mathrm{g}$ is the acceleration of gravity) at frequency sweeping, and the frequency range was set 5-20 $\mathrm{Hz}$.
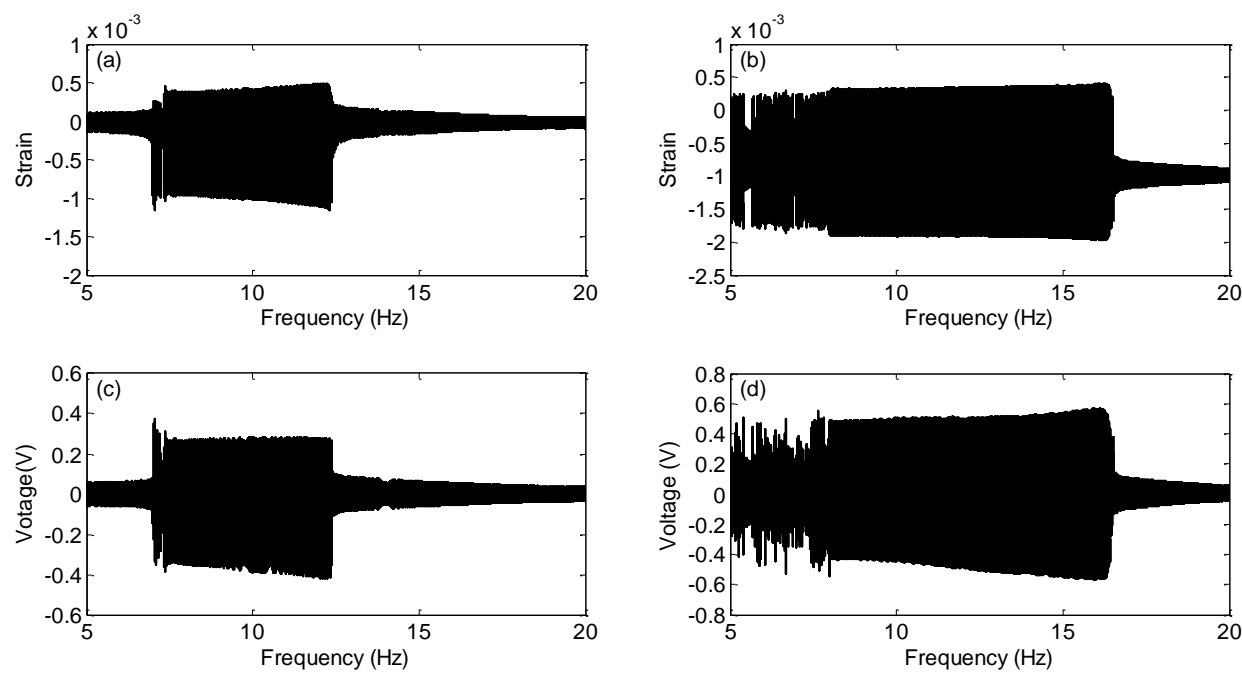

Fig. 12. Experiment strains and voltages at frequency sweeping excitation for $a_{b}=1.0 \mathrm{~g}$ : $(\mathrm{a}, \mathrm{c})$ $\mathrm{BEH} ;(\mathrm{b}, \mathrm{d}) \mathrm{QEH}$. 
Fig. 12 shows the measured strains and output voltages of the BEH and QEH. They are consistent with those of simulations in Fig. 9. It turns out that the QEH can keep snap-through over nearly a whole range of 5.0-16.7 Hz (Figs. 12(b) and 12(d)), whereas the BEH only has it within 7.2-12.3 Hz (Figs. 12(a) and 12(c)). As to output voltages, the QEH gives out higher voltages in a much wider bandwidth of frequency than the BEH. The highest voltage generated by the QEH can reach $0.52 \mathrm{~V}$, whereas that generated by the $\mathrm{BEH}$ is only $0.33 \mathrm{~V}$.
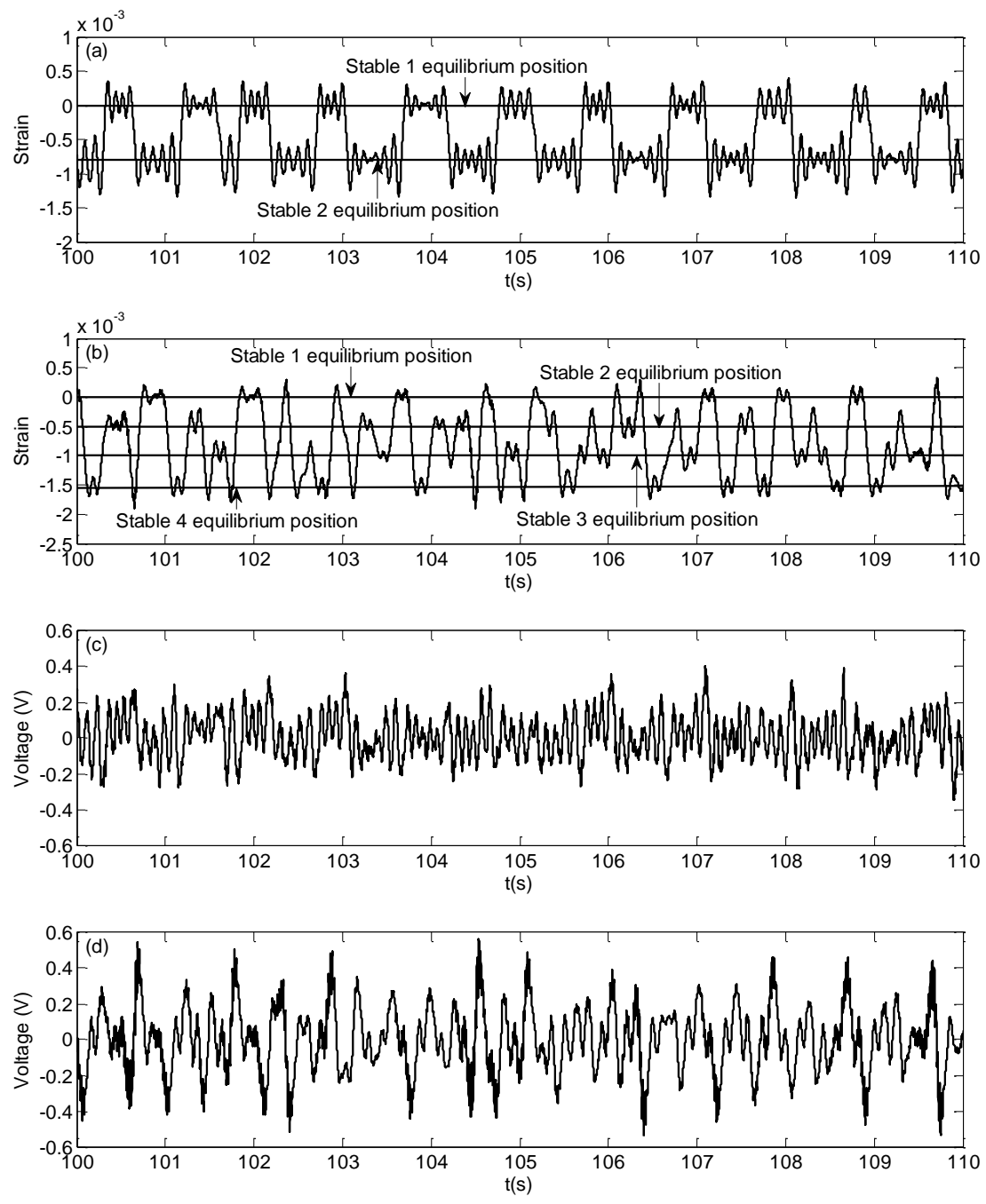

Fig. 13. Strains and voltages measured in experiment at snap-through: (a, c) BEH; (b, d) QEH.

To validate the snap-through characteristics of the $\mathrm{BEH}$ and $\mathrm{QEH}$, the excitation in experiment is set as $f=8 \mathrm{~Hz}$ and $a_{b}=1.0 \mathrm{~g}$, i.e., which is a harmonic excitation. The measured strains and voltages are shown in Fig. 13. It can be observed that the phenomena of snap-through are similar to the simulation results in Fig. 8. One can see that the response of the $\mathrm{BEH}$ needs to vibrate at an equilibrium position for a while before jumping to another (Fig. 13(a)). For the QEH, the snap-through occurs between 
four equilibrium positions continuously (Fig. 13(b)). Therefore, at snap-through the QEH can give out a dense voltage peak (Figs. 13(c) and 13(d)).

\section{Conclusion}

In this paper, we have developed a quad-stable piezoelectric device to harvest base vibration energy. By adjusting the three magnets on the fixture, the system can possess four stable equilibrium positions and become a quad-stable harvester. The potential energy and the electro-mechanical equation of the QEH are derived. Simulation results show that the QEH owns a smaller threshold and wider range of frequency for occurrence of snap-though than the BEH. The corresponding experiments are carried out for validation. The experimental results are in good agreement with the simulations. Therefore, it can be concluded that the presented quad-stable energy harvester is preferable to the bi-stable one in harvesting vibration energy.

\section{Acknowledgements}

The authors would like to thank the supports of the National Science Foundation of China (Grant No. 11172234).

\section{Funding}

The National Science Foundation of China (Grant No. 11172234).

\section{References}

[1] A. Erturk, D.J. Inman, Issues in mathematical modeling of piezoelectric energy harvesters, Smart Mater. Struct. 17 (2008) 065016.

[2] H. Vocca, I. Neri, F. Travasso, L. Gammaitoni, Kinetic energy harvesting with bistable oscillators, Appl. Energ. 97 (2012) 771-776.

[3] S. Roundy, P.K. Wright, A piezoelectric vibration based generator for wireless electronics, Smart Mater. Struct. 13 (2004) 1131-1144.

[4] A. Erturk, D.J. Inman, An experimentally validated bimorph cantilever model for piezoelectric energy harvesting from base excitations, Smart Mater. Struct. 18 (2009) 025009.

[5] K.A. Cook-Chennault, N. Thambi, A.M. Sastry, Powering MEMS portable devices-a review of non-regenerative and regenerative power supply systems with emphasis on piezoelectric energy harvesting systems, Smart Mater. Struct. 17 (2008) 043001.

[6] S. Roundy, Y. Zhang, Toward self-tuning adaptive vibration based micro-generators, Proc. SPIE 5649 (2005) 373-384. 
[7] S. Roundy, E. Leland, J. Baker, E. Carleton, E. Reilly, E. Lai, B. Otis, J. Rabaey, P. Wright, Improving power output for vibration-based energy scavengers, IEEE Pervas. Comput. 4 (2005) 28-36.

[8] J. Scruggs, An optimal stochastic control theory for distributed energyharvesting networks, J. Sound Vib. 320 (2009) 707-725.

[9] G. Litak, M.I. Friswell, S. Adhikari, Magnetopiezoelastic energy harvesting driven by random excitations, Appl. Phys. Lett. 96 (2010) 214103.

[10] M.F. Daqaq, Transduction of a bistable inductive generator driven by white and exponentially correlated Gaussian noise, J. Sound Vib. 330 (2011) 2554-2564.

[11] R. Ramlan, M.J. Brennan, B.R. Mace, I. Kovacic, Potential benefits of a non-linear stiffness in an energy harvesting device, Nonlinear Dyn. 59 (2010) 545-558.

[12] M.F. Daqaq, On intentional introduction of stiffness nonlinearities for energy harvesting under white Gaussian excitations, Nonlinear Dyn. 69 (2012) 1063-1079.

[13] A. Triplett, D. Quinn, The effect of non-linear piezoelectric coupling on vibration-based energy harvesting, J. Intell. Mater. Syst. Struct. 20 (2009) 1959-1967.

[14] M. Daqaq, C. Stabler, Y. Qaroush, T.S. Osorio, Investigation of power harvesting via parametric excitations, J. Intell. Mater. Syst. Struct. 20 (2009) 545-557.

[15] V.R. Challa, M.G. Prasad, Y. Shi, F.T. Fisher, A vibration energy harvesting device with bidirectional resonance frequency tenability, Smart Mater. Struct. 17 (2008) 015035.

[16] A. Erturk, D.J. Inman, Broadband piezoelectric power generation on high-energy orbits of the bistable Duffing oscillator with electromechanical coupling, J. Sound Vib. 330 (2011) 2339-2353.

[17] L. Tang, Y. Yang, C.K. Soh, Improving functionality of vibration energy harvesters using magnets, J. Intell. Mater. Syst. Struct. 23 (2012) 1433-1449.

[18] K. Miso, D. John, B.L. Wardle, Efficiency of piezoelectric mechanical vibration energy harvesting, Smart Mater. Struct. 24 (2015) 055006.

[19] S.P. Pellegrini, N. Tolou, M. Schenk, J.L. Herder, Bistable vibration energy harvesters: a review, J. Intell. Mater. Syst. Struct. 24 (2013) 1303-12.

[20] J.T. Lin, B. Lee, B. Alphenaar, The magnetic coupling of a piezoelectric cantilever for enhanced energy harvesting efficiency, Smart Mater. Struct. 19 (2010) 045012.

[21] S. Zhou, J. Cao, A. Erturk, J. Lin, Enhanced broadband piezoelectric energy harvesting using rotatable magnets, Appl. Phys. Lett. 102 (2013) 173901.

[22] L. Tang, Y. Yang, A nonlinear piezoelectric energy harvester with magnetic oscillator, Appl. Phys. Lett. 101 (2012) 094102.

[23] Y.J. Gao, Y.G. Leng, S.B. Fan, Z.H. Lai, Performance of bistable piezoelectric cantilever vibration energy harvesters with an elastic support external magnet, Smart Mater. Struc. 23 (2014) 095003. 
[24] J. Jung, P. Kim, J. Lee, J. Seok, Nonlinear dynamic and energetic characteristics of piezoelectric energy harvester with two rotatable external magnets, Int. J. Mech. Sci. 92 (2015) 206-222.

[25] P. Kim, J. Seok, A multi-stable energy harvester: dynamic modeling and bifurcation analysis, J. Sound Vib. 333 (2014) 5525-5547.

[26] S. Zhou, J. Cao, D.J. Inman, J. Lin, S.S. Liu, Z.Z. Wang, Broadband tristable energy harvester: modeling and experiment verification, Appl. Energ. 133 (2014) 33-39.

[27] S. Zhou, J. Cao, J. Lin, Z.Z. Wang, Exploitation of a tristable nonlinear oscillator for improving broadband vibration energy harvesting, Eur. Phys. J. Appl. Phys. 67 (2014) 30902.

[28] J. Cao, S. Zhou, W. Wang, J. Lin, Influence of potential well depth on nonlinear tristable energy harvesting, Appl. Phys. Lett. 106 (2015) 173903.

[29] G.T.O. Tékam, C.A.K. Kwuimy, P. Woafo, Analysis of tristable energy harvesting system having fractional order viscoelastic material, Chaos 25 (2015) 013112.

[30] S.C. Stanton, C.C. McGehee, B.P. Mann, Nonlinear dynamics for broadband energy harvesting: investigation of a bistable piezoelectric inertial generator, Physica D 239 (2010) 640-653.

[31] K. Yung, P. Landecker, D. Villani, An analytic solution for the force between two magnetic dipoles, Magn. Electric. Separat. 9 (1998) 39-52.

[32] H. Wu, L. Tang, C. K. Soh. Development of a broadband nonlinear two-degree-of-freedom piezoelectric energy harvester. Journal of Intelligent Material Systems and Structures, 25 (2014) 1875-1889.

[33] Y. G. Leng, Y. J. Gao, D. Tan, S. B. Fan, and Z. H. Lai, An elastic-support model for enhanced bistable piezoelectric energy harvesting from random vibrations, J. Appl. Phys. 117 (2015) 064901. 